Review Article

\title{
NANOPARTICLE INSULIN DRUG DELIVERY-APPLICATIONS AND NEW ASPECTS
}

\author{
PRIYANKA DAS ${ }^{*}$ \\ Department of pharmaceutics, Girijananda Chowdhury Institute of Pharmaceutical, Science, Hattkhowapara, Azara, Guwahati 781017 \\ Email: priyanz9321@gmail.com
}

Received: 10 Nov 2020, Revised and Accepted: 13 Jan 2021

\begin{abstract}
Diabetes mellitus is a chronic metabolic disorder caused via the deficiency of pancreatic hormone insulin (Type1 diabetes mellitus) or due to the resistance of cells to insulin secreted by using the body (Type 2 diabetes mellitus). It is a rapidly growing serious situation that inspires a lot of global concern. Exogenous insulin administration is many times used therapy for Type 1 Diabetes Mellitus and gestational diabetes. The traditional subcutaneous insulin injections cause a lot of suffering to the affected person, exceptionally due to pain and secondarily due to dose sensitivity and in additional complications. Hence alternate delivery systems are an area of recreation for medical professionals and a convenient alternative system will be a boon to the patients. As an end result of the technological advances, various invasive and non-invasive delivery systems have been brought in the previous years. Nanotechnology, particle-mediated delivery, pulmonary delivery, buccal spray, etc. are the most current advances.
\end{abstract}

Keywords: Diabetes, Insulin, Oral delivery, Insulin inhalers, Buccal delivery, Transdermal delivery, Insulin devices

(C) 2021 The Authors. Published by Innovare Academic Sciences Pvt Ltd. This is an open access article under the CC BY license (https://creativecommons.org/licenses/by/4.0/) DOI: https://dx.doi.org/10.22159/ijcpr.2021v13i2.41542. Journal homepage: https://innovareacademics.in/journals/index.php/ijcpr

\section{INTRODUCTION}

Diabetes Mellitus is a chronic disease of impaired glucose metabolism characterized by using hyperglycemia with altered carbohydrates, fats, and protein metabolism. It is of two types. Type 1 Diabetes Mellitus which is due to the atrophy of pancreatic $\beta$ cells, motives inadequate insulin secretion and as a result leaves the affected person completely dependent on exogenous insulin supply for his survival; and type 2 diabetes mellitus, in which peripheral cells grow to be resistant to the insulin secreted via the patient's body. Selective individuals with type 2 diabetes mellitus or these at a later stage of type 2 diabetes mellitus require exogenous insulin supply. The hormonal adjustments for the duration of being pregnant motivate glucose intolerance in some women. This is brought on due to the resistance of peripheral cells to insulin. This situation is referred to as gestational diabetes mellitus and this situation commonly reverses after delivery. In a few cases, this circumstance persists for a lifetime.4 Insulin therapy, which is the exogenous supply of insulin, is highly effective in regulating blood glucose levels in diabetes patients. DM reasons considerable rates of morbidity and mortality to the current population [1].

Insulin is used to manage the level of blood glucose in sufferers with DM. It is a quintessential therapy for patients suffering from T1DM, and T2DM (especially in late-stage disease). Insulin was once discovered in 1921 by Frederick Banting and Charles Best, went through its first scientific use in 1922 [2-4], and helped revolutionize the cure of T1DM, which was once deadly at that time. Initially, insulin was once isolated from bovine and porcine pancreata, till the 1980s. Later on, recombinant DNA strategies allowed the manufacture of human insulin. Modifications of the amino acid sequence of the insulin molecule using rDNA and protein engineering strategies have currently allowed the manufacturing of monomeric insulin analogs (e. g. lispro, aspart) which have a more rapid absorption profile [3].

Since DM results in a defect in insulin function, the perfect therapy is to permit diabetics to regain normal insulin function. However, present-day research and technology have no longer been capable to obtain this. The present-day insulin therapy includes exogenous administration to accomplish effective glycaemic manage (i.e. prevention of hyper-and hypoglycemia) and avoidance of the issues of DM [5]. Current modes of delivering insulin include intravenous (IV) infusion and subcutaneous (SC) injections. SC insulin preparations, which are greater typically used, include rapid-, intermediate-and long-acting insulin, which are used in extraordinary combos (1 to 4 times or more daily) [3].

Disadvantages of presently authorized insulin regimens relate to the injectable mode of its administration. Daily injections have led to non-compliance, which has apparent effects on patient outcomes. Also, parenteral administration bypasses the liver, unlike physiologically-secreted insulin. Hepatic first-pass metabolism, which decreases the quantity of circulating insulin via about $50 \%$ in normal individuals, prevents peripheral hyperinsulinemia and its related complications [5-11].

Delivery route of insulin

\section{Oral delivery}

Insulin remedy is correctly used in the therapy of diabetes mellitus. Insulin is a key participant in decreasing blood glucose levels for type 1 diabetes and is additionally required at later stages in type 2 diabetes patients. The extensively common route for delivery of insulin is through parenteral administration however, this delivery of insulin usually requires at least three or 4 daily insulin injections for good glycemic control. Consequently, more acceptable distinctive routes of insulin delivery have been searched to minimize suffering from discomfort, local pain, irritation, infection, immune reactions, and lipoatrophy at the injection website of insulin. Oral delivery of insulin would deliver the drug directly into the liver via portal circulation and ought to mimic the physiological destiny of endogenously secreted insulin [12-14]. However, polypeptides, like insulin, are degraded in the stomach $\mathrm{pH}$ and undergo proteolysis using enzymes in the gastrointestinal tract $[15,16]$. Moreover, the gastrointestinal mucosa has low permeability for large hydrophilic peptides.

To overcome the issues related to parenteral administration of insulin numerous techniques that are primarily based on nanotechnology has been developed to decorate the intestinal absorption of different protein and peptides. NPS consist of naturally taking place biodegradable polymers is extensively investigated in this regard. They have emerged as potential carriers of various therapeutic agents for managed drug delivery as well as the oral route of insulin. Various natural hydrophilic and hydrophobic polymers used as the carrier of oral insulin such as chitosan, alginate, dextran sulfate, etc. are normally used to prepare NPs.

\section{Polymers used as media for oral insulin delivery}

Over the previous few decades, improving interest has been paid to the use of polymeric NPs, either hydrophilic or hydrophobic as 
carriers for insulin delivery. Hydrophilic polymers are of unique activity due to their non-toxic, biocompatible, biodegradable, and herbal polymers. Among them, chitosan is extensively used due to the fact of its ease of chemical change and promising biological properties.

\section{Hydrophilic polymers}

\section{Chitosan (CS)}

CS is a well-known naturally occurring copolymer of beta-linked and $\mathrm{N}$-acetyl glucosamine and has been generally found in crustacean (crabs, shrimps, and lobsters) shell and some fungi or yeast. It is a biodegradable, biocompatible, non-toxic, non-allergic, easily absorbable natural hydrophilic polymer properties that have resulted in a wide array of applications in biomedical and drug delivery research $[17,18]$. It has also been shown as a paracellular permeability enhancer by interacting with the TJ proteins occluding ZO-1 and opens the tight junctions between epithelial cells $[19,20]$. In addition to these properties, it increases the stability of nanospheres and facilitates effective encapsulation of proteins and drugs that make it a suitable carrier material [21, 22]. CS has been extensively used to develop new chitosan derivatized polymers. In vivo, preclinical research of this formulation at a dose of $30 \mathrm{IU} / \mathrm{kg}$ in streptozotocin (STZ) induced diabetic rat models confirmed extended intestinal absorption of insulin from $\gamma$-PGA NPs. It has obtained a long-lasting hypoglycemic effect and $15 \%$ relative bioavailability in contrast to subcutaneous (sc) injection [23]. The identical system filled in enteric-coated capsules used to be even higher at the identical dose, showing $20 \%$ oral bioavailability. Also, as section insulin (=monomeric, 3 times quicker than regular) is encapsulated in the equal CS- $\gamma-\mathrm{PGA}$; it has got $15.7 \%$ oral bioavailability [24].

Moreover, insulin-loaded NPs with carboxylated chitosan and Polymethyl methacrylate (PMMA) had been developed to enhance insulin delivery through the oral route. One of the most broadly investigated polymers in the direction of peptide delivery is acrylates which have excessive interest due to the fact of its $\mathrm{pH}$ sensitivity and carboxyl groups to enhance the bioadhesive, alter the tight junction, chelate the $\mathrm{Ca} 2+$ thereby inhibiting the proteolytic activity of proteases, etc. They evaluated their capacity to decrease blood glucose levels in diabetic rats. In vivo experiments resulted in the reduction of blood glucose levels through $67 \%$ at a dose of 100 $\mathrm{IU} / \mathrm{kg}$ and the pharmacological bioavailability of the $25 \mathrm{IU} / \mathrm{kg}$ at a dose of PMMA NPs was once $9.7 \%[25,26]$.

Chitosan with sodium alginate is being organized as another insulin loaded nanoparticle product which is used to enhance the loading ability and activity maintenance. It's observed that when insulinloaded nanospheres $(25,50,100 \mathrm{IU} / \mathrm{kg})$ administered orally to diabetic rats they decreased glycemia in a dose-dependent manner. Their pharmacological availabilities are found 7.1, 6.8 and $3.4 \%$, respectively $[27,28]$.

\section{Dextran sulfate-vitamin B12}

Dextran sulfate is a non-toxic and highly water-soluble distinctive polymer used as matrices for oral delivery of insulin. Vitamin-B12 is proven as a ligand to enhance the uptake of the dextran NPs and their translocation throughout the gastrointestinal tract for excessive bioavailability. Insulin conjugated to dextran-vitamin B12 NPs to diabetic rats that had the least quantity of cross-linking was most effective at reducing blood glucose levels (70-75\%) in STZ brought on diabetic rats. Also, the hypoglycemic impact lasted for 54 h. This amendment confirmed the best hypoglycemic effect with a pharmacological availability of $29.4 \%[29,30]$.

\section{Hydrophobic polymers}

\section{Poly (lactide-co-glycolide) (PLGA)}

Particles consisting of PLGA have been extensively studied as therapeutic delivery vehicles owing to their biodegradable and biocompatibility particles. The hydrophobic nature of PLGA matrices typically makes them incapable of entrapping water-soluble insulin. Intragastric administration of the insulin-loaded PLGA NPs $(20$ $\mathrm{IU} / \mathrm{kg}$ ) to diabetic rats decreased fasting plasma glucose levels to
$57.4 \%$ inside the first $8 \mathrm{~h}$ of administration. The relative bioavailability of insulin following oral administration of NPS used to be $7.7 \%$ in contrast to the subcutaneous injection of its solution. Star-branched PLGA ( $\beta$-cyclodextrin-PLGA) NPs are highly promising for mitigating the burst impact and prolonging the release of insulin. Another study attempted to prevent the burst release of insulin in the stomach with the aid of the usage of a cellulose derivative (hydroxypropyl methylcellulose phthalate, HPMCP) to prepare PLGA NPs. This modification decreased the preliminary release of PLGA NPs in simulated gastric fluid from $50 \%$ to $20 \%$, and their relative bioavailability in diabetic rats was once approximately $6.2 \%[31,32]$.

\section{Polylactide acid (PLA)}

PLA showcases a strong affinity towards the small intestine due to their polyethylene oxide (PEO) blocks and an excessive permeation functionality towards the cell membrane owing to their amphiphilic property. When orally treated with vesicular PLA NPs loaded with insulin to diabetic mice (50 IU/ $\mathrm{kg}$ ), the highest blood glucose discount was once achieved at $4.5 \mathrm{~h}$. Although this effect lasted at least an extra $18.5 \mathrm{~h}$, growing the insulin concentration to $100 \mathrm{IU} / \mathrm{kg}$ did no longer enhance this hypoglycemic effect (hypoglycemic effect lasted for $23 \mathrm{hr}$ ) [33].

\section{Poly- $\varepsilon$-caprolactone (PCL)}

NPS organized with PCL and a monomeric form of insulin analog (aspart-insulin). Their outcomes demonstrated that this method permits for the protection of biological activities of insulin, an expansion of serum insulin levels, and enhancement of the glycemic response. The most impact of a reduction in hyperglycemia used to be found at $8 \mathrm{~h}$ after oral administration, which used to be more pronounced with aspart-insulin-loaded NPs (52\%) at the dose of 50 $\mathrm{IU} / \mathrm{kg}[34]$.

\section{Lipidic polymers}

\section{[Solid lipid NPS (SLN)]}

Previous research has demonstrated that nanoencapsulation of proteins in SLNs prolongs their blood residence time, modifies their redistribution, and improves their bioavailability [35]. Oral insulin delivery with SLNs administered to diabetic rats; their relative pharmacological bioavailability used to be $5.1 \%$ in contrast to SC injection of insulin; a considerable hypoglycemic effect used to be additionally observed during $24 \mathrm{~h}$. To facilitate the transport of particles throughout the cellular barriers, in another study, the relative bioavailability extended to $7.1 \%$.

\section{Insulin inhalers}

It is a non-invasive, well-tolerated delivery system, which is positive for each type 1 and types 2 diabetes mellitus. The glycaemic control via this approach is related to the subcutaneous route and it additionally enhances patient satisfaction, quality of life, and acceptance for Intense Insulin Therapy in a diabetic patient7. The aerosolized insulin has a diameter of about $3 \mu \mathrm{m}$, which enhances the alveolar disposition and low oropharyngeal and giant airway disposition. The onset of action following systemic absorption is about $20 \mathrm{~min}$, which is rapid. The action lasts for about 6-8 h, which is related to that in subcutaneous administration. Exubera was once the first inhaled insulin education available. It was once delivered with an aerosol device known as an Exubera inhaler. Nebulizers, metered-dose inhalers, and aqueous mist inhalers are being investigated. Inhalation is a remarkable mode for delivering premeal time insulin. It can be used for delivering fast-acting insulin only. It is much less wonderful in people who smoke and these with pulmonary diseases. The incidence of hypoglycemia is additionally elevated with this route [36,37].

\section{Buccal delivery}

Mucosal membranes of the internal lining of the cheeks can act as remarkable sites for insulin delivery. The area is robust, rich in blood supply, has expansive smooth muscle, and gives brief cellular restoration following injury or injury. Visibility and accessibility of buccal mucosa additionally make it the best site for delivery. The 
insulin sprayed into buccal mucosa can't enter deep lungs due to the fact of its size and subsequently, it is secure for lungs. 9 Insulin which is administered through the buccal route, is known as buccal insulin when it reaches the systemic circulation.1 The most important drawback of this route is the decreased bioavailability due to the extraordinarily low passage of active agents throughout the mucosal epithelium. Bioadhesive polymers can be used as an alternative. They adhere to the biological substrate to provide continued contact of the agent with the site of delivery. The quite several bioadhesive formulations consist of gels, films, tablets, vesicles, nanoparticles, and sponges. They are retained for a longer time and as a result, exhibit improved pharmacokinetic as well as absorption properties. Transferosomes Gels, patches, pelleted nanoparticles, tablets, sprays, films, sponges, etc. are the presently accessible buccal delivery formulations $[38,39]$.

\section{Pulmonary delivery}

Insulin that can be administered via a pulmonary route the usage of two methods-aerosol inhalation and intratracheal instillation. Aerosol presents a more uniform distribution with a greater extent of penetration into the peripheral or into the alveolar region of the lungs [40]. When delivered into the lungs, they are readily absorbed via the alveolar region directly into blood circulation. This can be completed via liquid nebulizers, aerosol-based metered dose inhalers, and dry powder dispersion devices. The simplicity of selfadministration, the large surface area of lungs that improves absorption, exceptionally excessive bioavailability, and noninvasiveness are the benefits of this delivery system [39].

\section{Insulin devices}

Insulin infusion devices may also be categorized as open-loop and closed-loop systems. Programmable open-loop micropump insulin delivery system consists of a small, lightweight, transportable insulin micropump and plastic tubing that connects the pump to a needle inserted below the skin. Insulin release patterns in them can be preprogrammed and initiated by using the timer or via the diabetic affected person himself. This device needs very careful monitoring of blood glucose levels. Also, the patients using these devices have been suggested to exhibit an excessive incidence of ketoacidosis. Implantable variations of open-loop insulin infusion units have been additionally introduced. Chemically managed closed-loop insulin delivery devices work by way of a remarks mechanism. It is an effective alternative in the absence of an effective pancreas or $\beta$-cell transplantation. It mimics pancreatic activity. They are biocompatible and non-toxic. The biohybrid artificial pancreas is any other type of insulin diffusion device, which is beneath research. These contain $\beta$-cells enclosed inside a semipermeable membrane, which is biocompatible. The semi-permeable membrane is permeable to glucose and insulin. Special has to be given to apart from immune cells to forestall rejection utilizing the body [41-43].

\section{Transdermal delivery}

Transdermal insulin delivery is an attractive choice to the invasive parenteral route of administration and different alternative routes of insulin such as pulmonary and nasal routes due to the fact the skin provides the benefits of easy access and a very massive surface area. It improves patient compliance and avoids each liver's first-pass metabolism and the degradation of drugs in the gastrointestinal tract. The pores and skin additionally represent a vital painless interface for systemic drug administration. Despite these benefits, the human pores and skin limit the permeation of foreign compounds particularly large hydrophilic molecules like insulin. The stratum corneum; which is the top layer, reasons the impermeability of the skin by way of its lipid-rich matrix. Several attempts have been made to overcome the pores and skin barrier and to enable the transfer of large drugs such as insulin. They can be divided into chemical (liposome and chemical enhancers) and physical techniques (mainly iontophoresis and sonophoresis).

\section{Transdermal delivery methods}

Chemical enhancers such as surfactants, fatty acids, fatty esters, and azone-like compounds alter the lipid shape of the stratum corneum.
They minimize its barrier properties and enhance its permeability for massive molecule drugs that would not pass via the skin.

Iontophoresis is a non-invasive approach used to enlarge transdermal insulin penetration through the pores and skin by using the application of a small electric current potential. Large drug molecules can be delivered in a shorter time with the assist of this technique and it will increase drug mobility.

Another non-invasive method sonophoresis (ultrasound, phonophoresis) which has been used to enhance (and or delivery and activity of drugs) pores and skin permeability to a number low and high molecules weight drugs such as inulin. Low-frequency ultrasound $(20-160 \mathrm{kHz})$ decreases blood glucose levels both in animal and human studies [44-46].

Microneedles are a minimally invasive, painless and promising technology to deliver drugs into the skin barring disruption of nerve endings. This technology creates micron-sized channels in which interstitial fluid fills up the channels in the skin. It makes the hydrophilic transport pathway, allows the stratum corneum barrier, and will increase skin permeability to giant molecules $[44,46]$.

\section{Vaginal delivery}

In current years numerous studies show that vagina has received rich blood supply and massive surface area that means good permeability and can be a practicable route for systemic delivery to a broad range of compounds. The essential benefits of vaginal drug routes are avoidance of the first-pass metabolism, ease of administration, and exact permeability for low molecular weight drugs. For systemic delivery bile salts, dihydro fusidate, cyclodextrins, surfactants, and chelating agents have been examined as enhancers to facilitate the rate of vaginal absorption however sometimes; they caused several local reactions $[44,46]$.

\section{Rectal delivery}

Rectal route of delivery has been examined quickly after the discovery of insulin however, several investigators have met absorption problems via the mucosa. This administration's promising benefit is the opportunity of avoiding, to some extent, the hepatic first-pass metabolism. Absorption promoters and surfactants had been used to furnish the highest hypoglycemic effect in rectal insulin delivery. The most effective rectal absorption enhancer polyoxyethylene-9-lauryl ether (POELE) or sodium salicylate, was once used in insulin suppositories on diabetic dogs. It was once investigated that the hypoglycemic effect can be achieved via about $50-55 \%$ [47].

\section{Insulin devices using pen devices}

It is a convenient and correct technique for insulin delivery. It intends to enhance glycemic control by making it much less difficult to comply with the present-day recommendations for intensive insulin regimens. Two sorts of pens are available; prefilled and reusable. Pens are available in various styles. Insulin pens have the potential to become an essential asset for elevated compliance amongst all patients undergoing insulin therapy.

\section{Other novel strategies in insulin delivery}

Erythrocytes, which are the most abundant cells in the body, can be used as tremendous carriers of many different drugs together with insulin. Biocompatibility, biodegradability, long circulation half-life, and the potential to get loaded with a variety of chemically and biologically active compounds make resealed erythrocytes outstanding carriers of therapeutic agents [48]. Dendrimers are macromolecules with highly branched 3D structures [49]. They additionally are used for the successful delivery of insulin.

\section{CONCLUSION}

The research and developments in insulin delivery are really promising. Many problems make the patient hesitant to provoke insulin therapy. So, many alternative routes for insulin delivery are beneath research and some of them are available. Each one has its benefits as well as drawbacks. An appropriate alternative, with high effectivity and good patient compliance, is probably to emerge in the 
future to help out people with type1 diabetes, gestational diabetes, and type 2 diabetes not managed with oral hypoglycaemic agents.

\section{FUNDING}

Nil

\section{AUTHORS CONTRIBUTIONS}

All the authors have contributed equally.

\section{CONFLICTS OF INTERESTS}

Declared none

\section{REFERENCES}

1. Jaleh Varshosaz. Insulin delivery systems for controlling diabetes-recent patents on endocrine. Metab Immune Drug Discovery 2007;1:25-40.

2. Vaughan M, Hill RL. The discovery of insulin: the work of frederick banting and charles best. J Biol Chem 2002;277:e15.

3. Fisher M, Frier BM. Diabetes mellitus in Davidson's principles and practice of medicine; 2006 .

4. Rosenfeld L. Insulin: discovery and controversy. Clin Chem 2002:48:2270-88.

5. Yaturu S. Current and future trends at dawn: insulin therapies. World J Diabetes 2013;4:1-7.

6. Chen KJ, Chen MC, Sung HW, Sonaje K. A review of the prospects for polymeric nanoparticle platforms in oral insulin delivery. Biomaterials 2011;32:9826-38.

7. Garmel G, Martinez R. Gas gangrene from subcutaneous insulin administration. Am J Emerg Med 1993;11:622-5.

8. Lin CK, Sonaje K, Lin KJ, Yeh TH. Biodistribution, pharmacodynamics, and pharmacokinetics of insulin analogs in a rat model: oral delivery using $\mathrm{pH}-$-responsive nanoparticles vs. subcutaneous injection. Biomaterials 2010;31:6849-58.

9. Arbit E. The physiological rationale for oral insulin administration. Diabetes Technol Ther 2004;6:510-7.

10. Chowdhury EH, Ahmad A, Zaini A, Othman I. Oral nano-insulin therapy: current progress on nanoparticle-based devices for intestinal epithelium-targeted insulin delivery. J Nanomed Nanotechnol 2012:S4-007. DOI:10.4172/2157-7439.S4-007

11. Bargman JM. Intraperitoneal versus subcutaneous insulin in patients on nighttime IPD. Adv Perit Dial 1994;10:116-9.

12. Carvalho RA, Woitiski CB, Veiga F, Neufeld RJ, Figueiredo IV. Pharmacological effect of orally delivered insulin facilitated $\mathrm{b}$ multilayered stable NPs. Eur J Pharm Sci 2010;41:556-63.

13. Woitiski CB, Carvalho RA, Sarmento B, Veiga F, Neufeld RJ. Facilitated nanoscale delivery of insulin across intestinal membrane models. Int J Pharm 2011;412:123-31.

14. Nature S. Insulin therapies: current and future trends at dawn. World J Diabetes 2013;4:1-7.

15. Zhi ZL, Khan F, Birch DJS, Saxl T. Nanomedicine and it's potential in diabetes research and practice. Diabetes/Metab Res Rev 2008;24:604-10.

16. Lassmann Vague V, Raccah D. Alternatives routes of insulin delivery. Diabetes Metab 2006;32:513-22.

17. Avadi MR, Mohammadpour N, Abedin S, Atyabi F, Sadeghi AMM. Preparation and characterization of insulin NPs using chitosan and Arabic gum with ionic gelation method. Nanomed: Nanotechnol Biol Med 2010;6:58-63.

18. Sonaje K, Hsu LW, Chuang EY, Liao ZX, Chuang EY. pHResponsive NPS shelled with chitosan for oral delivery of insulin. Mechanism Ther Appl Chem Res 2012;45:619-29.

19. Jintapattanakit A, Kissel T, Mao S, Zetterberg J, Bakowsky U, Junyaprasert VB. Peroral delivery of insulin using chitosan derivatives: A comparative study of polyelectrolyte nanocomplexes and NPs. Int J Pharma 2007;342:240-9.

20. Lim LY, Ma Z, Lim TM. Pharmacological activity of peroral chitosaninsulin NPs in diabetic rats. Int J Pharm 2005;293:271-80.

21. Kundu PP, Mukhopadhyay P, Rana D, Mishra R. Strategies for effective oral insulin delivery with modified chitosan NPs. Progress Polymer Sci 2002;37:1457-75.

22. Jin Y, Song Y, Zhou D, Zhang Z, Zhu X, Chen C. Goblet celltargeting NPS for oral insulin delivery and the influence of mucus on insulin transport. Biomaterials 2012;33:1573-82.
23. Sonaje $\mathrm{K}$, Lin $\mathrm{YH}$, Juang JH, Wey SP, Chen $\mathrm{CT}$, Sung HW. In vivo evaluation of safety and efficacy of self-assembled NPs for oral insulin delivery. Biomaterials 2009;30:2329-39.

24. Sonaje K, Nguyen HN, Hsu CW, Chen YJ, Chen HL, Wey SP, Juang $\mathrm{JH}$. Enteric-coated capsules filled with freeze-dried chitosan/poly( $\gamma$-glutamic acid)NPs for oral insulin delivery. Biomaterials 2010;31:3384-94.

25. Sajeesh S, Ponchel G, Sharma CP, Vauthier C, Gueutin C. Thiol functionalized polymethacrylic acid-based hydrogel microparticles for oral insulin delivery. Acta Biomater 2010;6:3072-80.

26. Tang C, Yin C, Cui F, Qian F, Zhao Z, Yin L. Preparation, characterization, and oral delivery of insulin-loaded carboxylated chitosan grafted poly(methyl methacrylate) NPs. Biomacromolecules 2009;10:1253-8.

27. Woitiski CB, Ribeiro A, Neufeld R, Veiga F. Design for optimization of NPS integrating biomaterials for orally dosed insulin. Eur J Pharm Biopharm 2009;73:25-33.

28. Veiga F, Ferreira D, Sarmento B, Riberio A. Development and characterization of new insulin-containing polysaccharide NPs. Colloids Surfaces 2006;53:193-202.

29. Chalasani KB, Jain AK, Diwan PV, Jain SK, Russell Jones GJ. Effective oral delivery of insulin in animal models using vitamin B12-coated dextran NPs. J Controlled Release 2007;112:14150.

30. Chalasani KB, Yandrapu SK, Diwan PV, Russell Jones GJ. A novel vitamin B12-nanosphere conjugate carrier system for peroral delivery of insulin. J Controlled Release 2007;425:421-9.

31. Wu ZM, Qian Y, Luo KQ, Zhang LJ, Zhou L, Guo XD, et al. HP55coated capsule containing PLGA/RS NPs for oral delivery of insulin. Int J Pharm 2012;425:1-8.

32. Cun DM, Zhang LQ, Cui FD, Tao AJ. Preparation of insulinloaded PLGAHP55NPS for oral delivery. J Pharma Sci 2007;96:421-7.

33. Xiong XY, Zhou CL, Tam KC, Li YP, Li ZL. Vesicles from pluronic/poly(lactic acid) block copolymers as new carriers for oral insulin delivery. J Controlled Release 2007;120:11-7.

34. Ubrich N, Damge C, Maincent P. Oral delivery of insulin associated with polymeric NPS in diabetic rats. J Controlled Release 2007;117:163-70.

35. Liu J, Zhang Z, Gong T, Zhong Z, Wang C. Solid lipid NPs loaded with insulin by sodium cholate-phosphatidylcholine-based mixed micelles: Preparation and characterization. Int J Pharm 2007;340:153-62.

36. Rajiv Dhand, Vamsi P. Guntur-inhaled insulin: extending the horizons of inhalation therapy. Respiratory Care 2007;52:911-22.

37. S Chauhan Nitesh, Singh Vijender, Chauhan Sanjeev, Handa Vandana. Recent advances in insulin delivery systems: an update. World Appl Sci J 2010;11:1552-6.

38. R Kumria, G Goomber. Emerging trends in insulin delivery; buccal route. J Diabet 2011;2:1-8.

39. Jaleh Varshosaz. Insulin delivery systems for controlling diabetes-recent patents endocrine. Metab Immune Drug Discovery 2007;1:25-40.

40. Patel Binal, R Pandey Shivanand, NP Jivan, Choudhary Amruta, Mahalakshmi, Devmurari Viral. Pulmonary delivery as a route for insulin. Int J PharmTech Res 2009;1:1190-7.

41. Todd C Zion, Jackie Y Ying, Henry H Tsang. Glucose-sensitive nanoparticles for controlled insulin delivery. Available from: https://hdl.handle.net/1721.1/3783 [Last accessed on 05 Oct 2020].

42. Valerie Ravaine, Bogdan Catargi, Christophe Ancla. Chemically controlled closed-loop insulin delivery. J Controlled Release 2008;132:2-11.

43. AH Clemens. Programmable open loopmicropump insulin delivery system. Diab Care 1980;3:359-61.

44. Lassmann Vague V, Raccah D. Alternatives routes of insulin delivery. Diabetes Metabol 2006;32:513-22.

45. Garcia Contreras L, Hickey AJ, Morçöl T, Bell SJD. Evaluation of novel particles as pulmonary delivery systems for insulin in rats. AAPS PharmSciTech 2003;5:1.

46. Sharma JPK, Bansal S, Banik A. Noninvasive routes of proteins and peptides drug delivery. Indian J Pharm Sci 2011;73:367-75. 
47. Hosny E, Al-Shora HI, Elmazar MM. Relative hypoglycemic effect of insulin suppositories in diabetic beagle dogs: optimization of various concentrations of sodium salicylate and polyoxyethylene-9-lauryl ether. Biol Pharm Bull 2001;24:1294-7.
48. Shashank Shah. Novel drug delivery carrier: resealed erythrocytes. Int J Pharm Biosci 2001;2:394-406.

49. Tarun Garg, Onkar Singh, Saahil Arora, RSR Murthy. Dendrimer a novel scaffolding for drug delivery. Int J Pharm Sci Rev Res 2011;7:211-20 